Audiology

Neurotology
Audiol Neurotol 2015;20:172-182

DOI: 10.1159/000369608
Received: April 8, 2014

Accepted after revision: November 4, 2014

Published online: April 8, 2015

\title{
Selecting Appropriate Dose Regimens for AM-101 in the Intratympanic Treatment of Acute Inner Ear Tinnitus
}

\author{
Hinrich Staecker $^{a} \quad$ Kenneth S. Maxwell ${ }^{b} \quad J o h n$ R. Morris ${ }^{c}$ Paul van de Heyning ${ }^{d}$ \\ Krzysztof Morawski ${ }^{e} \quad$ Frank Reintjes ${ }^{f}$ Thomas Meyer ${ }^{g}$ \\ a Department of Otolaryngology, Head and Neck Surgery, University of Kansas Medical Center, Kansas City, Kans., \\ bPiedmont Ear, Nose and Throat Associates, Winston-Salem, N.C., and ' Advanced ENT and Allergy, Louisville, Ky., \\ USA; ${ }^{d}$ Department of ENT, Head and Neck Surgery, Antwerp University Hospital, University of Antwerp, Antwerp, \\ Belgium; ${ }^{e}$ Department of Otolaryngology, Medical University of Warsaw, Warsaw, Poland; ${ }^{\mathrm{f}} \mathrm{HNO}$ Praxis im \\ Schlosscarree, Braunschweig, Germany; ${ }^{9}$ Auris Medical AG, Basel, Switzerland
}

\section{Key Words}

Tinnitus $\cdot$ Hearing loss - Acute acoustic trauma .

Barotrauma Surgery trauma $\cdot$ Otitis media $\cdot$ Cochlea . Intratympanic therapy $\cdot \mathrm{AM}-101 \cdot \mathrm{N}$-methyl-D-aspartate receptor

\begin{abstract}
Inhibition of cochlear N-methyl-D-aspartate (NMDA) receptors with AM-101, a small molecule antagonist delivered by intratympanic injection, represents a novel approach to treat acute tinnitus triggered by glutamate excitotoxicity. An earlier double-blind, randomized, placebo-controlled phase II clinical trial (TACTTO) had demonstrated a significant and dose-dependent improvement in tinnitus triggered by acute acoustic trauma or otitis media from baseline to day 90. A second phase II trial (TACTT1) now sought to evaluate the most appropriate dose regimen for this treatment. Outcomes from the TACTT1 trial showed no significant difference in tinnitus improvement between a single-dose treatment and a dose regimen comprising three doses over 2 weeks. Taken together, three injections over 3 consecutive days showed the best results in the two phase II trials, suggesting that repeated and concentrated inhibi-
\end{abstract}

tion of cochlear NMDA receptors provides best treatment effects, while keeping the procedural impact on patients short.

(c) 2015 S. Karger AG, Basel

\section{Introduction}

Tinnitus, the perception of sound without external acoustic stimulation, is one of the most common disorders seen in otolaryngology practices. About 16 million US adults are estimated as having frequent tinnitus in a given year [Shargorodsky et al., 2010], with a survey showing a prevalence of severely annoying tinnitus in $1.6 \%$ of the adult population [Baguley et al., 2013]. Tinnitus may seriously impact the ability to sleep or relax, or lead to tiredness, irritation, nervousness, despair, frustration or depression [Stouffer and Tyler, 1990; Chan, 2009]. However, there is no universally accepted standard of care for tinnitus or approved tinnitus drug [Langguth and Elgoyhen, 2012], provoking substantial frustration among patients and physicians [Hall et al., 2011].

Various attempts have been made to treat peripheral tinnitus with intratympanically delivered drugs [Meyer,

\begin{tabular}{ll}
\hline KARGER 125: & $\begin{array}{l}\text { (C) 2015 S. Karger AG, Basel } \\
\text { 1420-3030/15/0203-0172\$39.50/0 Open access }\end{array}$ \\
$\begin{array}{l}\text { E-Mail karger@karger.com } \\
\text { www.karger.com/aud }\end{array}$ & $\begin{array}{l}\text { This is an Open Access article licensed under the terms of the } \\
\text { Creative Commons Attribution-NonCommercial 3.0 Un- } \\
\text { ported license (CC BY-NC) (www.karger.com/OA-license), } \\
\text { applicable to the online version of the article only. Distribu- } \\
\text { tion permitted for non-commercial purposes only. }\end{array}$
\end{tabular}

Thomas Meyer, $\mathrm{PhD}$

Auris Medical AG

Falknerstrasse 4

$\mathrm{CH}-4001$ Basel (Switzerland)

E-Mail ear@aurismedical.com 
2013]. Intratympanic (i.t.) lidocaine, in spite of early promise, is rarely used for tinnitus management nowadays due to unacceptable side effects and only transient efficacy [Dodson and Sismanis, 2004]. The small-molecule drug AM-101 (esketamine hydrochloride gel; Auris Medical AG, Basel, Switzerland) represents a new approach to the treatment of peripheral tinnitus in its acute stage before it may become centralized at higher structures of the auditory system. AM-101 is delivered intratympanically in a biocompatible hyaluronic acid gel and blocks cochlear N-methyl-D-aspartate (NMDA) receptors. These have been proposed as the source of aberrant excitation of the auditory nerve following glutamate excitotoxicity [Puel et al., 2002], which can be triggered by traumatic or vascular incidents [Pujol and Puel, 1999], or may be provoked by proinflammatory cytokines [Hwang et al., 2011].

Clinical development of AM-101 was initiated with a phase I/II study, which enrolled 24 patients suffering from acute tinnitus following acute acoustic trauma (AAT) or idiopathic sudden sensorineural hearing loss (ISSNHL) [Muehlmeier et al., 2011]. Single doses of AM101 were well tolerated up to the maximum tested concentration of $0.81 \mathrm{mg} / \mathrm{ml}$, and only small traces of the active substance and its primary metabolite could be detected in plasma, confirming the minimal systemic exposure afforded by the i.t. approach.

This was followed by a phase II clinical trial (TACTT0) which enrolled 248 patients suffering from tinnitus following AAT, ISSNHL or acute otitis media. Trial participants received 3 i.t. injections of either AM-101 0.27 or $0.81 \mathrm{mg} / \mathrm{ml}$ or placebo over 3 consecutive days. The trial showed for AAT- or otitis media-triggered tinnitus a statistically significant, dose-dependent and nonreversible improvement in tinnitus loudness, annoyance, sleep difficulties and general tinnitus impact [van de Heyning et al., 2014]; e.g. the reduction in tinnitus loudness from baseline to the last follow-up visit at day 90 in the AM-101 high-dose group reached 26.7 points or $48 \%$ versus 5.0 points or $9 \%$ in the placebo group. This represents an effect size of 0.88 (defined as difference in absolute improvement between AM-101 and placebo divided by the average of their respective standard deviation), which is considered large according to Cohen's rule of thumb [Meikle et al., 2008]. Patients presenting with unilateral tinnitus showed better responses than bilateral cases, who, as a precaution, were treated only unilaterally. The patient subgroup with ISSNHL-related tinnitus did not show conclusive results due to an unexpectedly high rate of spontaneous remission and possibly inadvertent inclusion of cases with noncochlear etiologies.

AM-101 Dose Regimens in Acute Inner Ear Tinnitus
The TACTT1 trial ('Comparison of Single versus Repeat Doses of AM-101 in the Treatment of Acute Inner Ear Tinnitus') was designed to further evaluate the appropriate dosing regimen for AM-101, notably by testing single-dose applications and a triple-dose regimen stretching over 2 weeks instead of 3 days. One of the key questions for the study to address was whether repeated doses were better than a single dose in attenuating tinnitus or not, and whether longer intervals between doses influenced treatment effect sizes. The eligible patient population was broadened to include two other types of traumatic tinnitus triggers, barotrauma and middle ear surgery. Also, bilateral treatment was introduced for bilateral tinnitus cases, based on the favorable safety outcomes from the TACTT0 trial. For efficacy analysis, the outcome measures were mostly retained. Instead of the minimum masking level (MML), which had turned out to be highly variable, patient-reported tinnitus loudness was selected as the primary efficacy variable. Tinnitus loudness provides a direct, though subjective measure of a key symptom characteristic [Tyler et al., 2007; Landgrebe et al., 2012].

\section{Methods}

The TACTT1 trial was designed as a multicenter, double-blind, randomized, placebo-controlled exploratory phase II trial with two sequential dose cohorts. Cohort 1 evaluated a single-dose regimen and cohort 2 assessed a triple-dose regimen. The trial involved 5 sites in the USA and 11 sites in Europe (Germany, Belgium, and Poland). The trial was registered on ClinicalTrials. gov (NCT 01270282) and conducted in compliance with the Declaration of Helsinki, the relevant guidelines of the International Conference on Harmonisation and Good Clinical Practice. The clinical trial was approved by the relevant institutional review boards/ethics committees as well as by the FDA and corresponding European health authorities.

Eligible participants were aged 18-65 years, had persistent tinnitus following a documented incident of AAT, inner ear barotrauma, middle ear surgery, acute otitis media or ISSNHL with onset no later than 3 months prior to randomization. Early in the trial, ISSNHL-related tinnitus was excluded by protocol amendment upon finding inconclusive results for this etiology in the TACTT0 trial. Exclusion criteria included fluctuating or intermittent tinnitus, Ménière's disease, ongoing acute or chronic otitis media or externa, any ongoing therapy known as potentially tinnitus-inducing or any therapy for hearing loss or otitis media, concomitant use of any other NMDA receptor antagonist or of other tinnitus treatments. Breast-feeding and pregnant women or women of childbearing potential who were unwilling or unable to practice an effective method of contraception were excluded. Written informed consent was obtained from each patient prior to the performance of any study-specific procedures. 


\section{Randomization and Masking}

Patients were randomized to receive AM-101 $0.81 \mathrm{mg} / \mathrm{ml}$ or placebo (vehicle only) at a 2:1 ratio. The active and placebo formulations had the same appearance and viscosity and revealed no differences during or following administration. The study drug was provided to study sites in identical kits and sequentially numbered with an identifier for the study site and one for each individual patient. A separate randomization sequence was generated for each study site. Patients and investigators remained blinded to study drug allocation throughout the trial.

\section{Procedures}

For each patient the trial consisted of a baseline assessment on the first treatment visit (TV1; day 0), two additional treatment visits on day 7 (TV2) and day 14 (TV3) in cohort 2, and 3 followup visits which were scheduled 7, 30 and 90 days after the (last) treatment (FUV1-FUV3). Baseline assessments included a general physical examination, vital signs, blood sampling, a urine drug test, and a urine pregnancy test for women of childbearing age. Tinnitus was considered persistent when patients stated that they could always hear their tinnitus when they were thinking of it in the past 2 weeks and that their tinnitus during that period occurred either all of the time, most of the time or a good bit of the time.

At all study visits, subjective tinnitus loudness 'right now' (TLQ) and tinnitus annoyance 'today' (TAQ) were determined on a 0 - to 100 -point numerical rating scale. The absolute improvement of TLQ from baseline to FUV3 was defined as primary efficacy endpoint. At all study visits the MML was determined at $1 \mathrm{~dB}$ resolution by using the ascending method of limits and pulsed broadband masking noise as well as the tinnitus loudness match. The latter was determined against a pulsed $1-\mathrm{kHz}$ tone [Vernon and Meikle, 2003] at $2 \mathrm{~dB}$ resolution. Both psychoacoustic measures were performed 3 times and results averaged.

The Tinnitus Handicap Questionnaire (THQ) [Kuk et al., 1990] was administered at baseline and FUV3. Item 11 of the questionnaire ('My tinnitus has gotten worse over the years') was omitted since it was not applicable to acute tinnitus. Besides the total score, item 5 ('I have trouble falling asleep at night because of tinnitus') was analyzed separately as it relates to one of the most frequent complaints of tinnitus sufferers [Stouffer and Tyler, 1990]. The Patient Global Impression of Change in tinnitus severity from baseline was queried at all follow-up visits. Response categories included very much better, much better (jointly representing pronounced improvement), minimally better, unchanged, minimally worse (jointly no or minor changes) and much worse or very much worse (together pronounced worsening).

Pure-tone audiometry $(0.25-8 \mathrm{kHz})$ was performed at all study visits using the ascending method of limits with results recorded on audiograms. The primary safety endpoint was the frequency of clinically relevant hearing deterioration in the treated ear, defined as threshold shift $\geq 15 \mathrm{~dB}$ from baseline to FUV2 at the average of any two contiguous test frequencies. Further safety assessments included evaluation for the presence of spontaneous nystagmus, otoscopy and tympanometry.

The study drug was administered by i.t. injection after the tympanic membrane had been locally anesthetized. Approximately $0.25 \mathrm{ml}$ was gently injected into the middle ear following myringotomy with the patient's head placed in a position tilted $45^{\circ}$ towards the untreated ear. Patients remained in their reclined or su- pine position for approximately $30 \mathrm{~min}$ to allow the active substance to diffuse into the cochlea. In case of bilateral tinnitus the second ear was treated no later than 90 min after the first ear.

For pharmacokinetic evaluations 5-ml blood samples were collected from the first 18 patients of cohort 1 prior to treatment administration on day 0 and at 15,30, 45, 60, 180 and 360 min after treatment. Samples were prepared and analyzed as previously described [Muehlmeier et al., 2011].

\section{Statistical Methods}

This exploratory clinical trial was intended to obtain data for two different dose regimens. Hence no statistical hypotheses were defined for confirmatory testing, but the trial was expected to indicate relevant efficacy trends. For exploratory comparison of single-dose AM-101, triple-dose AM-101 and placebo group means (the latter pooled from both cohorts), 24 patients per group were determined sufficient for comparison of the three group means using a contrast for trend in the one-way analysis of covariance (ANCOVA) at a significance level of 0.05 . This resulted in a sample size of 72 evaluable patients for the entire trial ( 36 per cohort). The two placebo groups in each cohort were pooled based on the assumption that the number of treatments did not have any impact on efficacy or safety outcomes; however, placebo data were also analyzed by cohort, and the appropriateness of pooling was assessed statistically.

Efficacy analyses were performed on a modified 'intention-totreat' analysis set, which included all patients who received all required study medication, had TLQ values at baseline and FUV3, and no major protocol violations. Missing values were not imputed. In case of bilateral treatment, efficacy outcome measures from both ears were averaged. Safety analyses were performed for the 'safety population' analysis set, which included all patients who received the study drug at least once. For safety analyses, cohort 2 patients receiving only one injection were assigned to cohort 1 , while those receiving 2 injections instead of 3 remained in cohort 2.

For continuous efficacy endpoints, ANCOVA models were used including treatment group as fixed class effect, the baseline values of the respective endpoint as covariate and a planned comparison (contrast for trend). Based on an expected relative improvement in TLQ from baseline to FUV3 (placebo: 0, AM-101 single dose: 10 points, AM-101 triple dose: 15 points) contrasts of $-5,1$, and 4 were used. In addition, comparisons of the treatment groups were performed pairwise as well as by cohort between AM101 and placebo. For the discrete endpoint Patient Global Impression of Change, the Cochran-Mantel-Haenszel test for trend was applied. The frequency of patients meeting the primary safety endpoint was compared by active group and corresponding placebo group with the Fisher exact test.

For comparison of the TACTT1 results with those from a comparable study population in the TACTT0 trial a forest plot was generated [Lewis and Clarke, 2001] based on a fixed effect model. Analyzed were all patients with unilateral tinnitus related to AAT or otitis media and treated with AM-101 $0.81 \mathrm{mg} / \mathrm{ml}$. Effect sizes were calculated for each dose regimen - triple dose over 3 consecutive days, single dose, and triple dose over 2 weeks - as standardized mean difference between the improvement in TLQ by FUV3 in the AM-101 group and its corresponding placebo group. They were weighted by the inverse of the variance of the effect size for calculation of the combined effect. 


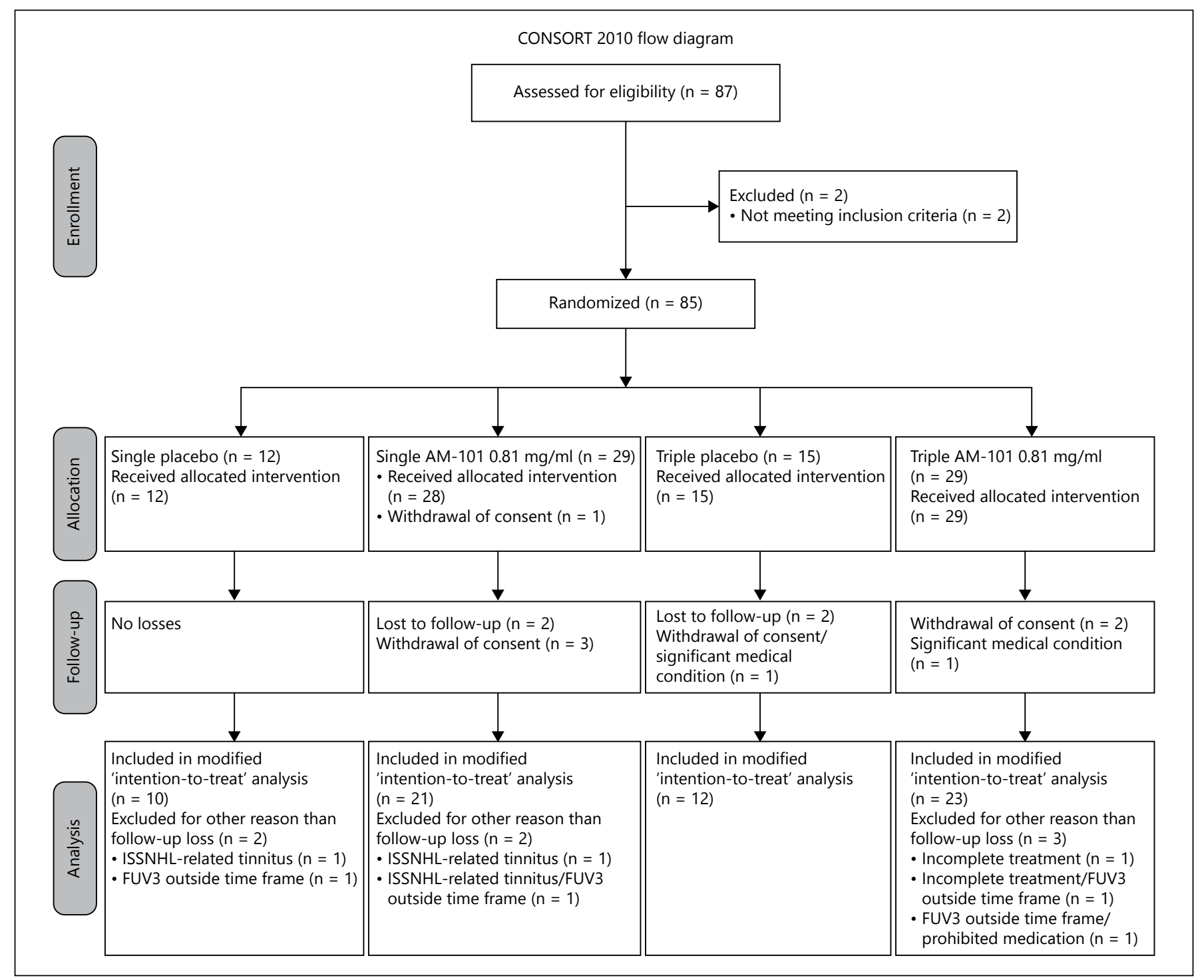

Fig. 1. Patient flow chart for the TACTT1 trial in accordance with the CONSORT 2010 statement [Moher et al., 2001]. In cohort 1, 1 randomized patient declined treatment (AM-101 $0.81 \mathrm{mg} / \mathrm{ml})$. In cohort 2, 8 patients did not receive all of their planned injections ( $6 \times$ single-dose administration only and $2 \times$ two injections only);

\section{Results}

\section{Patient Demographics and Characteristics}

The trial profile in accordance with the CONSORT statement [Moher et al., 2001] is shown in figure 1. A total of 85 patients were randomized ( 41 into cohort 1, 44 into cohort 2), of which 84 were included in the Safety Population analysis set and 66 in the modified 'intentionto-treat' analysis set. The most common reasons for exclusion from the latter were incomplete treatment cycle in 2 of these cases, further administration was contraindicated due to otitis media or inflammation of the eardrum. Overall 7 patients withdrew consent, 1 patient was withdrawn by the investigator due to a significant medical condition (depression), and 4 patients were lost to follow-up. in cohort 2, FUV3 taking place outside the allowed time range, and use of prohibited concomitant medication. Two patients in the single AM-101 $0.81 \mathrm{mg} / \mathrm{ml}$ group and 1 patient in the single placebo group were excluded as their tinnitus was triggered by ISSNHL. Overall, 73 patients $(86 \%)$ completed the trial.

Baseline demographics and patient characteristics for the study population are presented in table 1 . The majority of patients were male (71\%), which is in line with previously reported data [Nicolas-Puel et al., 2006], and had 
Table 1. Patient demographics and characteristics at baseline

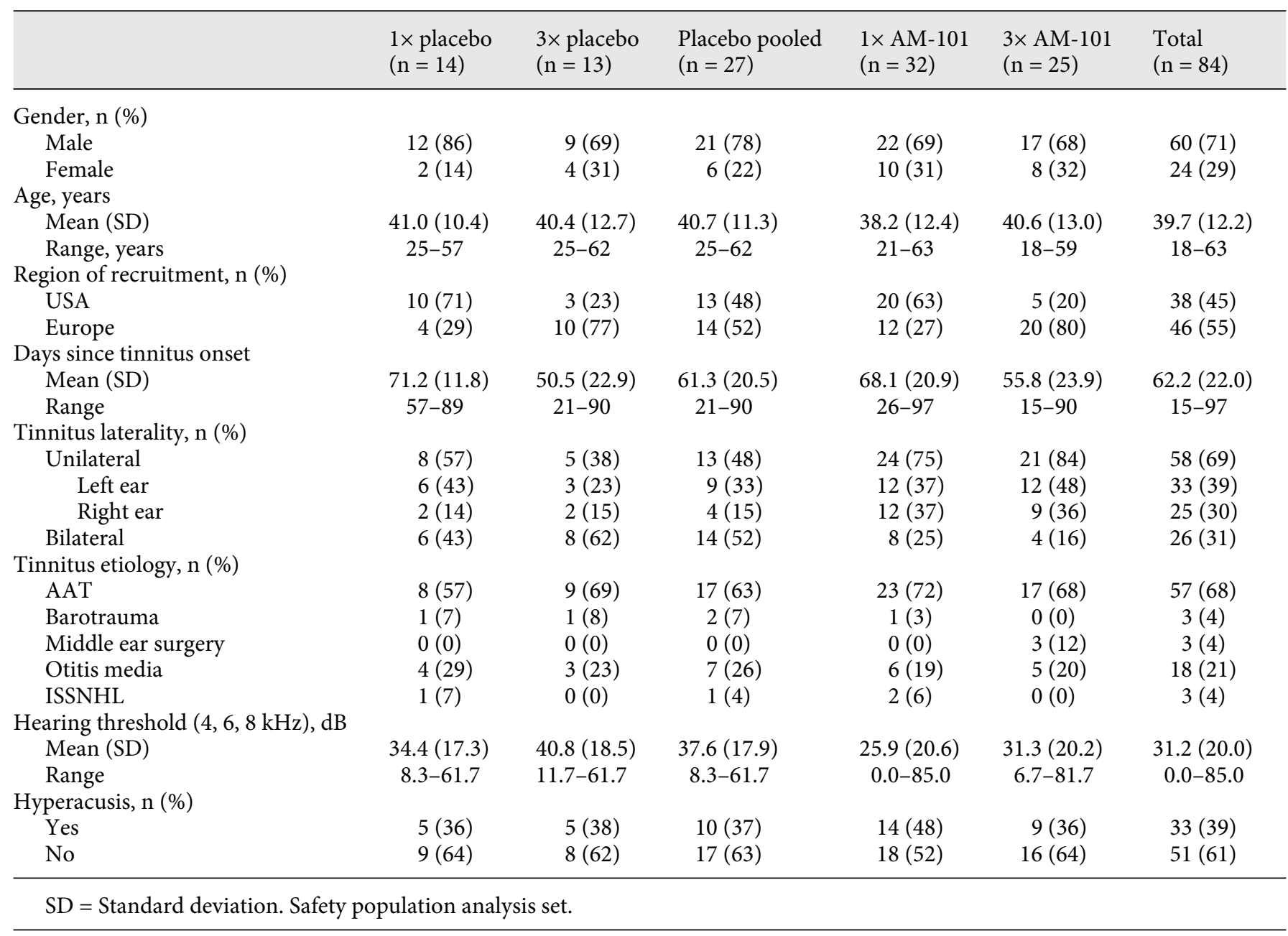

unilateral tinnitus (69\%). The most common tinnitus onset factors were AAT (68\%) and otitis media (21\%). Mean time since tinnitus onset was 62 days. Baseline values for tinnitus efficacy variables are presented in table 2. Overall, baseline demographics and characteristics were similar for the different cohorts and treatment groups.

\section{Efficacy Outcomes}

Descriptive statistics show improvement in the patientreported outcomes (PROs) for tinnitus loudness, annoyance, sleep difficulties and the THQ score from baseline to FUV3 for both dose regimens (table 3). TLQ shows gradual improvement starting at FUV1, the first observation point when eardrums are generally closed again, and continuing to FUV3 (fig. 2); the effect size was 0.5 for triple-dose AM101 and 0.4 for single-dose AM-101, i.e. small to moderate and small, respectively. The trend for the MML and tinnitus loudness match (table 3) was consistent with that of PROs for cohort 2, but not cohort 1 . Both variables showed high variability with a coefficient of variation of 1.9 or higher.

The ANCOVA model for the improvement in TLQ showed no statistically significant trend in group means for pooled placebo, single-dose AM-101 and triple-dose AM-101 ( $\mathrm{p}=0.084)$. Pairwise comparison with pooled placebo showed a statistically significant improvement for single-dose AM-101 ( $\mathrm{p}=0.044$; table 4$)$, but not for triple-dose AM-101 $(\mathrm{p}=0.286)$. Consistent with relative effect sizes, the treatment effect by cohort was higher for the triple dose than for the single dose (10.9 vs. 7.2 points).

A statistically significant global trend was observed for the improvement in sleep difficulties as measured by THQ item 5 ( $\mathrm{p}=0.007)$; the difference in least square means for single- and triple-dose AM-101 groups versus pooled placebo was statistically significant (18.8 and 13.9 
Table 2. Baseline values for efficacy variables

\begin{tabular}{|c|c|c|c|c|c|c|}
\hline \multicolumn{7}{|c|}{ Tinnitus loudness, TLQ } \\
\hline Mean (SD) & $48.8(25.8)$ & $51.7(20.5)$ & $50.3(22.5)$ & $52.9(23.6)$ & $49.0(18.1)$ & $50.7(21.2)$ \\
\hline Range & $15-85$ & $20-100$ & $15-100$ & $7-100$ & $15-80$ & $7-100$ \\
\hline \multicolumn{7}{|c|}{ Tinnitus annoyance, TAQ } \\
\hline \multicolumn{7}{|l|}{ THQ } \\
\hline Mean (SD) & $32.8(21.8)$ & $38.5(22.7)$ & $35.9(21.9)$ & $34.1(19.3)$ & $37.7(22.8)$ & $35.9(21.2)$ \\
\hline Range & $7.5-75.4$ & $11.9-81.2$ & $7.5-81.2$ & $7.7-64.5$ & $7.5-87.3$ & $7.5-87.3$ \\
\hline \multicolumn{7}{|c|}{ Difficulties falling asleep } \\
\hline Mean (SD) & $38.0(27.5)$ & $42.9(32.9)$ & $40.7(30.0)$ & $44.3(34.0)$ & $30.5(30.1)$ & $38.3(31.4)$ \\
\hline Range & $0-90$ & $0-90$ & $0-90$ & $0-100$ & $0-100$ & $0-100$ \\
\hline Mean (SD) & $29.0(27.4)$ & $20.4(15.0)$ & $24.4(22.2)$ & $15.3(8.9)$ & $21.0(17.9)$ & $20.0(16.4)$ \\
\hline Range & $1.7-84.3$ & $0.0-54.3$ & $0.0-84.3$ & $0.7-38.7$ & $1.7-65.0$ & $0.0-84.3$ \\
\hline
\end{tabular}

SD = Standard deviation; SL = sensation level. Difficulties falling asleep: THQ item 5. Modified 'intention-to-treat' analysis set.

Table 3. Change in efficacy variables from baseline to FUV3

\begin{tabular}{|c|c|c|c|c|c|}
\hline Mean improvement from baseline (SD) & $\begin{array}{l}1 \times \text { placebo } \\
(n=10)\end{array}$ & $\begin{array}{l}3 \times \text { placebo } \\
(n=12)\end{array}$ & $\begin{array}{l}\text { Placebo pooled } \\
(n=22)\end{array}$ & $\begin{array}{l}1 \times \text { AM-101 } \\
(n=21)\end{array}$ & $\begin{array}{l}3 \times A M-101 \\
(n=23)\end{array}$ \\
\hline Tinnitus loudness & $11.3(34.2)$ & $1.9(20.3)$ & $6.1(27.2)$ & $21.4(20.1)$ & $12.9(23.9)$ \\
\hline Tinnitus annoyance & $15.8(38.2)$ & $10.0(18.6)$ & $12.6(28.5)$ & $22.2(24.8)$ & $14.3(21.1)$ \\
\hline Difficulty falling asleep & $2.0(26.5)$ & $7.9(12.9)$ & $5.7(14.0)$ & $26.0(29.6)$ & $13.8(29.5)$ \\
\hline THQ total score & $3.3(15.5)$ & $7.8(12.9)$ & $5.7(14.0)$ & $12.9(11.4)$ & $11.5(17.9)$ \\
\hline MML & $5.6(17.3)$ & $3.3(6.1)$ & $4.3(12.1)$ & $1.8(11.8)$ & $5.2(13.0)$ \\
\hline
\end{tabular}

Mean improvement from baseline to FUV3 (SD = standard deviation). Modified 'intention-to-treat' analysis set.

points, $\mathrm{p}=0.005$ and 0.034 ). The THQ and TAQ showed also superior improvements for both AM-101 groups over placebo, but less pronounced: 7.7 and 5.2 points $(\mathrm{p}=$ 0.061 and 0.190 ) for THQ, and 10.5 and 4.4 points ( $\mathrm{p}=$ 0.138 and 0.523 ) for TAQ. The test for global trend showed $p$ values of 0.060 and 0.188 , respectively. In contrast, outcomes were not conclusive for the MML and tinnitus loudness match and not consistent with PRO trends.

In line with PROs, both single- and triple-dose AM101 groups showed a higher share of pronounced improvement in Patient Global Impression of Change in tinnitus severity compared with placebo from baseline to
FUV3 (fig. 3). The difference against pooled placebo was statistically significant $(\mathrm{p}=0.035$ and 0.029$)$ and also the difference in cohort $2(\mathrm{p}=0.036)$. The majority of placebo-treated patients reported no or only minor changes in severity. Overall, there were no cases of pronounced worsening in tinnitus over the trial duration.

Although there were differences in outcomes for the single- and triple-dose placebo groups, these were not consistent across all PROs (table 3); t tests for comparison of the PROs in an ANCOVA model with baseline values as covariates showed no statistically significant difference between the groups ( $p$ values $\geq 0.3$ ). 


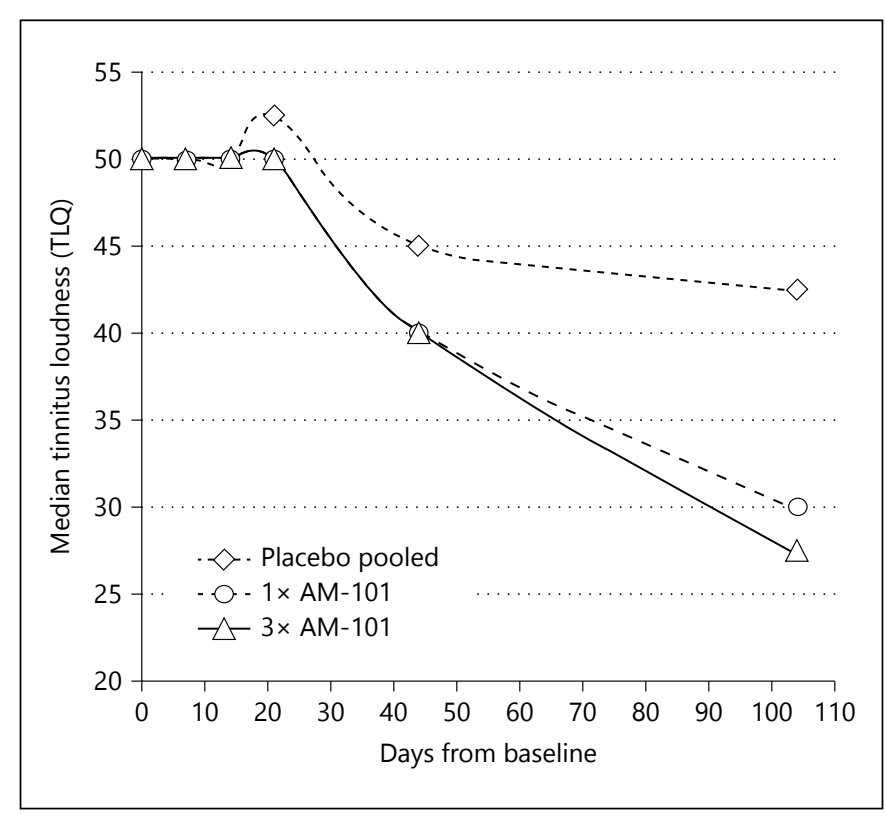

Fig. 2. Median tinnitus loudness ratings (TLQ) from baseline to FUV3. For comparison, values for pooled placebo and single-dose AM-101 are synchronized with the visit schedule for cohort 2, with values for the first 14 days set as stable. Modified 'intention-totreat' analysis set, $\mathrm{n}=66$.

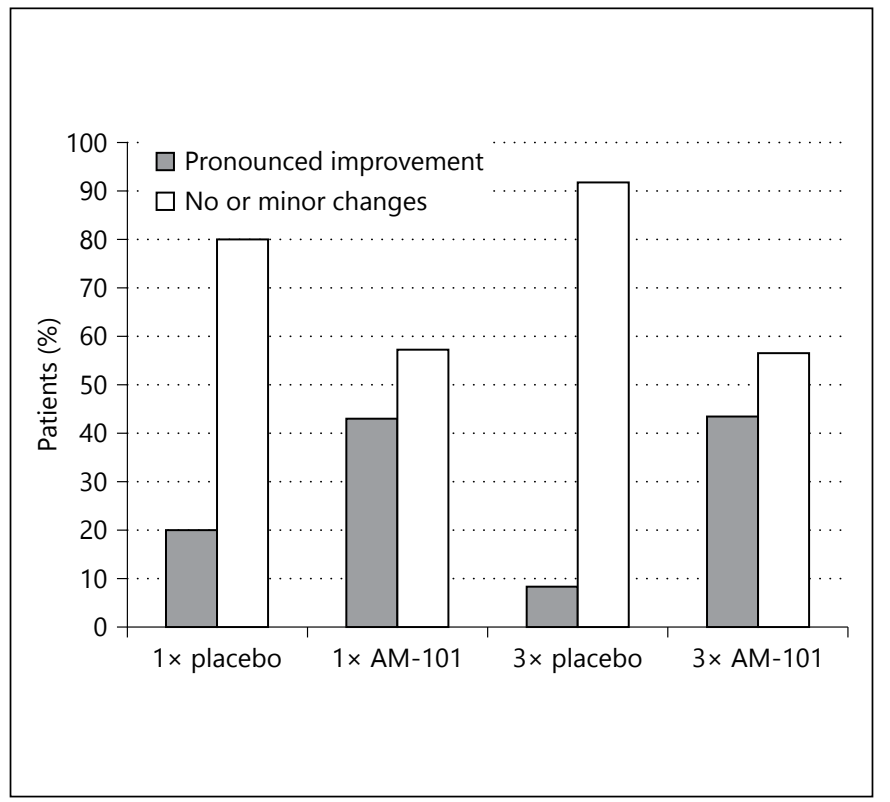

Fig. 3. Share of patients with pronounced improvement (much or very much better) or no or minor changes (minimally better, unchanged or minimally worse) in their Global Impression of Change in tinnitus severity from baseline to FUV3. There was no case of pronounced worsening. Modified 'intention-to-treat' analysis set, $\mathrm{n}=66$.

Table 4. ANCOVA results for improvement in tinnitus loudness to FUV3

$\begin{array}{lllll}\begin{array}{l}1 \times \text { placebo } \\ (\mathrm{n}=10)\end{array} & \begin{array}{l}3 \times \text { placebo } \\ (\mathrm{n}=12)\end{array} & \begin{array}{l}\text { Placebo pooled } \\ (\mathrm{n}=22)\end{array} & \begin{array}{l}1 \times \text { AM-101 } \\ (\mathrm{n}=21)\end{array} & \begin{array}{l}3 \times \text { AM-101 } \\ (\mathrm{n}=23)\end{array}\end{array}$

\section{Pairwise}

LS means, $\mathrm{n}$

6.27

20.53

13.54

LS mean difference

$-6.99(-6.73,20.70)$
$\mathrm{p}=0.312$
$14.26(0.42,28.10)$
$\mathrm{p}=0.044^{*}$
$7.27(-6.25,20.78)$
$\mathrm{p}=0.286$

\section{By cohort}

LS means, $\mathrm{n}$

13.24

1.97

20.46

12.82

LS mean difference

$7.21(-7.85,22.27)$

$$
\mathrm{p}=0.335
$$

$$
\begin{gathered}
10.85(-5.95,27.66) \\
p=0.198
\end{gathered}
$$

LS $=$ Least squares. $95 \%$ confidence intervals are given in parentheses. * Significant at 0.05 level. Modified 'intention-to-treat' analysis set. 


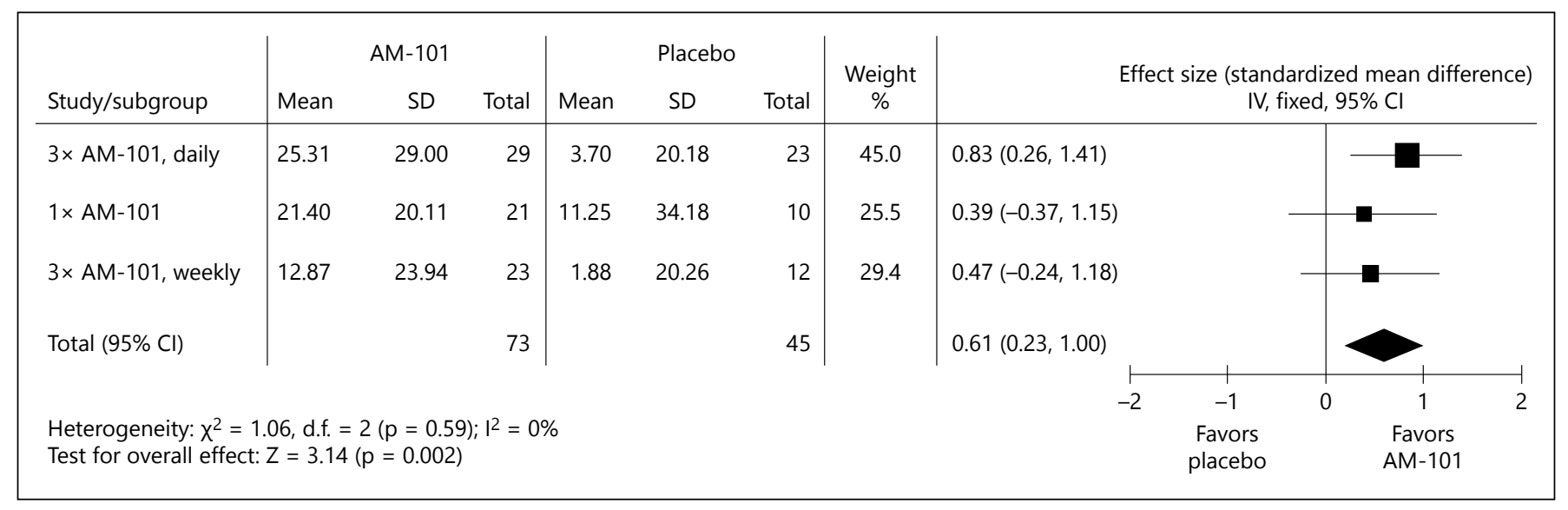

Fig. 4. Effect size (standardized mean difference) for improvement in tinnitus loudness from baseline to FUV3 in studies TACTT0 and TACTT1 determined with fixed effect model and presented as a forest plot. SD = Standard deviation; $\mathrm{IV}=$ inverse variance; $\mathrm{CI}=$ confidence interval.

Comparison of the improvement in tinnitus loudness from baseline to FUV3 across the TACTT0 and TACTT1 trials showed a statistically significant overall effect for treatment with AM-101 over placebo ( $\mathrm{p}=0.002$; fig. 4). The mean treatment effect (improvement in tinnitus loudness in active-treated patients, less improvement in placebo-treated patients) was 15.8 points (95\% confidence interval 6.7-25.0). This corresponds to an overall treatment effect size of 0.61 (95\% confidence interval $0.23-1.0)$, which is considered moderate. The test for heterogeneity was not significant $(\mathrm{p}=0.59)$, i.e. the differences observed between AM-101 and placebo were not fundamentally different among the three dose regimens. At 25.3 points, the daily treatment over 3 consecutive days showed the best absolute improvement and a large effect size $(0.83,95 \%$ confidence interval $0.26-1.41)$.

\section{Safety Outcomes}

Clinically relevant hearing deterioration at FUV2 was observed in 1 patient (single-dose AM-101; resolved). Cumulated over all visits, the frequency of occurrence was 0 and $18.5 \%$ for placebo and AM-101 in cohort 1 and 15.4 and $16.0 \%$ in cohort 2 . The difference in occurrence between AM-101 and placebo was statistically not significant. Most of the cases occurred around the injection procedure. Hearing thresholds at 4, 6 and $8 \mathrm{kHz}$ between baseline and FUV 3 remained essentially unchanged. At FUV1, the eardrum was closed in $68 \%$ of patients who received one injection and $87 \%$ of patients who received multiple injections. By FUV2 all eardrums were closed. No impact of the study drug on vestibular function was observed.
Adverse events (AEs) were reported by similar proportions of patients across the treatment groups with no apparent clinically relevant differences in frequency, intensity or relationship (table 5). No severe AEs were reported, and local events accounted for $>50 \%$ of reported AEs. They related mostly to anticipated transient changes in tinnitus perception and hearing arising from the myringotomy or were unrelated. Tinnitus was reported as louder in 7 instances before FUV1, which is to be attributed to the procedure; 4 of these cases resolved, 2 had an unknown outcome due to study discontinuation, and 1 remained unresolved (single placebo; moderate). In 6 cases the tinnitus AE occurred only after FUV1 or in the untreated ear, suggesting lack of causal relationship. The observed resolution of tinnitus AEs is in line with the aforementioned absence of profound deterioration in tinnitus severity from baseline to day 90 .

In each treatment group, there was one $\mathrm{AE}$ leading to study discontinuation: middle ear inflammation of moderate severity at TV2, rated as probably related by the investigator (triple placebo); depression of moderate severity at TV1, unlikely related (single AM-101; patient withdrawn by investigator); increase in tinnitus of mild severity from TV1 with resolution 3 days later, unlikely related (triple AM-101), and worsening of tinnitus of moderate severity at TV1, possibly related (single placebo). In 1 case (otitis media of mild severity from TV1 with resolution 14 days later, possibly related, triple AM-101), the study medication was discontinued, but the patient completed the study. Overall just one serious AE occurred (cubital tunnel syndrome of mild severity at day 30 , unrelated). 
Table 5. Most common AEs

\begin{tabular}{|c|c|c|c|c|c|c|}
\hline & $\begin{array}{l}1 \times \text { placebo } \\
(\mathrm{n}=14)\end{array}$ & $\begin{array}{l}3 \times \text { placebo } \\
(\mathrm{n}=13)\end{array}$ & $\begin{array}{l}\text { Placebo pooled } \\
(\mathrm{n}=27)\end{array}$ & $\begin{array}{l}1 \times \text { AM-101 } \\
(n=32)\end{array}$ & $\begin{array}{l}3 \times A M-101 \\
(n=25)\end{array}$ & $\begin{array}{l}\text { Total } \\
(\mathrm{n}=84)\end{array}$ \\
\hline Any AE & 24 & 17 & 41 & 34 & 38 & 113 \\
\hline \multicolumn{7}{|l|}{ Severity (all causality) } \\
\hline Mild & $6(43)$ & $5(39)$ & $11(41)$ & $12(38)$ & $9(36)$ & $32(38)$ \\
\hline \multicolumn{7}{|l|}{ Relatedness } \\
\hline Related & $4(29)$ & $2(15)$ & $6(22)$ & $4(13)$ & $8(32)$ & $18(21)$ \\
\hline Unrelated & $5(36)$ & $6(46)$ & $11(41)$ & $11(34)$ & $5(20)$ & $27(32)$ \\
\hline \multicolumn{7}{|l|}{ Most frequent AEs } \\
\hline Middle ear inflammation, otitis media & $0(0)$ & $1(8)$ & $1(4)$ & $1(3)$ & $0(0)$ & $2(2)$ \\
\hline Nasopharyngitis & $0(0)$ & $2(15)$ & $2(7)$ & $0(0)$ & $0(0)$ & $2(2)$ \\
\hline Hypercholesterolemia & $1(7)$ & $0(0)$ & $1(4)$ & $1(3)$ & $0(0)$ & $2(2)$ \\
\hline Blood triglycerides increased & $0(0)$ & $0(0)$ & $0(0)$ & $1(3)$ & $1(4)$ & $2(2)$ \\
\hline Back pain & $1(7)$ & $1(8)$ & $3(11)$ & $0(0)$ & $0(0)$ & $2(2)$ \\
\hline Dizziness & $1(7)$ & $0(0)$ & $1(4)$ & $0(0)$ & $1(4)$ & $2(2)$ \\
\hline Depression & $1(3)$ & $1(8)$ & $2(7)$ & $0(0)$ & $0(0)$ & $2(2)$ \\
\hline
\end{tabular}

Frequencies and percentages (in parentheses) of patients with AEs ( $>2 \%$ overall); occurrence of an AE in the same patient was counted only once. Safety population analysis set.

Bioanalysis showed a maximum concentration of the active substance in blood plasma $30 \mathrm{~min}$ after treatment at $<0.2 \mathrm{ng} / \mathrm{ml}$ and for the primary metabolite $3 \mathrm{~h}$ after treatment at $<0.15 \mathrm{ng} / \mathrm{ml}$.

\section{Discussion}

The current trial is the latest of three randomized controlled trials with AM-101 in the treatment of acute peripheral tinnitus. In total, almost 360 patients have been enrolled who received a total of 940 i.t. injections, of which more than 600 contained AM-101. The two phase II studies were conducted with essentially the same outcome measures and design and within comparable study populations - with the exception of the dose regimen and the exclusion of the challenging ISSNHL subpopulation in TACTT1. The trials can thus be easily compared.

Although the TACTT1 trial was exploratory and not powered to demonstrate statistical significance between treatment groups, the PROs showed a consistent trend for superior improvement over placebo for AM-101 groups. The improvement in sleep difficulties and the Patient Global Impression of Change in tinnitus severity were statistically significantly better for both active groups than for the placebo group; for tinnitus loudness, it reached significance in the single-dose group. Overall, outcomes for single-dose and triple-dose AM-101 were essentially similar, i.e. adding a second and third dose 7 and 14 days after the first one did not yield incremental benefits. As in the preceding trial, the psychoacoustic measures MML and loudness match did not show results that were consistent with PROs and were marked by high variability, putting their suitability as efficacy outcome measure further into question.

Treatment effects in TACTT1 were clearly smaller than in the previous trial, where AM-101 had been administered 3 times over 3 consecutive days. For example, in TACTT1 the treatment effect observed with tinnitus loudness was $10-11$ points on the $0-100$ rating scale for each of the AM-101 groups, whereas it was about twice that number in TACTT0. This observation suggests that 
repeated and concentrated application of AM-101 and hence concentrated inhibition of cochlear NMDA receptors provide superior treatment benefits.

In a separate evaluation of a responder criterion based on improvement in tinnitus loudness, a change of 20 points has been proposed with anchor-based, distribution-based and cumulative distribution approaches using pooled data from the two studies [DeMuro-Mercon et al., 2014]. Other authors have proposed a 10-point cutoff point as a minimal clinically identifiable difference [Adamchic et al., 2012]. With a 25.3-point absolute improvement the dose regimen comprising 3 i.t. injections over 3 days showed stronger evidence for clinical meaningfulness than single-dose treatment or 3 i.t. injections over 2 weeks (12.9-21.4 points).

The gradual improvement in PROs observed in the TACTT1 trial to the end-of-study visit 90 days after the last injection is in line with the results observed in the preceding, larger TACTT0 study. This pattern seems to suggest a lasting benefit - if the treatment effect was to wear off, it would do so more quickly than only after 3 months as small molecules such as the active substance of AM-101 tend to get cleared from the cochlea within a few days already [e.g. Chen et al., 2003; Salt, 2005; Bird et al., 2011]. The gradual improvement is most likely the result of adaptation processes within the auditory system [van de Heyning et al., 2014].

The TACTT1 trial confirmed the absence of effects of AM-101 on hearing or balance and of systemic side effects. The lack of systemic risks was further corroborated by the very low blood plasma concentrations that were comparable to previously reported results [Muehlmeier et al., 2011]. The i.t. administration was again accompanied by transient procedure-related side effects in a number of patients - notably an increase in tinnitus loudness and muffled hearing. These effects usually resolved with closure of the tympanic membrane. The triple-dose regimen over 2 weeks was tolerated as well as the single-dose regimen, and results were similar to outcomes from the previous trial (incidence of related AEs $13-32 \%$ in TACTT1 vs. $27-30 \%$ in TACTT0 [van de Heyning et al., 2014]). Bilateral treatment was generally well tolerated.

Compared to the previous trial, in which 5 days after the last injection only $7 \%$ of eardrums remained open, the TACTT1 trial revealed less favorable tympanic membrane closure rates. Whilst in the previous trial Xylocaine $10 \%$ pump spray had been used as local anesthetic, no particular anesthetic was specified for TACTT1. Phenol, the anesthetic of choice at US sites, and, more rarely, Bonain's solution (a mixture of phenol, cocaine and men- thol), were applied to $43 \%$ of patients. Since these two products were applied in $87 \%$ of the cases with open eardrums 1 week after injection, while delayed closure was observed only rarely with other anesthetics (Xylocaine, lignocaine, tetracaine or EMLA cream), it appears that phenol may not be ideal for patient comfort. Further, time to eardrum closure may also be of relevance for treatment effects as altered perception of sound and increased tinnitus may influence patient expectations.

In conclusion, the accumulated data suggest that a dose regimen of 3 i.t. injections over a short period of time provides superior treatment benefits for AM-101, while limiting the procedural impact on the patient. AM-101 appears to be a promising novel approach for the otolaryngologist to treat tinnitus mediated by glutamate excitotoxicity with a short and local therapy before the disorder becomes permanent and may require long-term management. Further clinical trials with AM-101 with a larger number of patients shall seek to confirm the phase II findings and notably demonstrate clinical relevance of a reduction in tinnitus loudness by a likewise reduction in tinnitus impact. In addition, it appears worthwhile exploring the treatment's efficacy further in tinnitus triggered by other insults than acute traumatic cochlear injury or otitis media or beyond the acute stage.

\section{Acknowledgments}

The authors wish to thank Dr. Richard S. Tyler, Iowa City, Iowa, USA, for his help and support in defining and specifying audiological assessments, and Dr. Manfred Wargenau, Dr. Frauke Friedrichs and Dr. Marcos Marin-Galiano, MARCO Institute, Düsseldorf, Germany, for their biostatistical contributions. Sincere gratitude is also expressed to all participating tinnitus patients who made this trial possible and to all investigators and their staff: USA: Patrick J. Antonelli, Gainesville, Fla., Jed A. Kwartler, Berkeley Heights, N.J., Kenneth S. Maxwell, Winston-Salem, N.C., John R. Morris, Louisville, Ky., Hinrich Staecker, Kansas City, Kans.; Belgium: Tony Cox, Hasselt, Ingeborg Dhooge, Gent, Paul van de Heyning and Sara Rabau, Antwerp; Germany: Marianne Grohé, Cologne, Florian Heimlich, Heidelberg, Guido Mühlmeier, Ulm, Frank Reintjes, Braunschweig, Sebastian Strieth, Frankfurt; Poland: Wojciech Domka, Rzeszów, Grazyna Lisowska, Tarnowskie Góry, Krzysztof Morawski, Warsaw.

\section{Disclosure Statement}

The clinical trial was supported in full by Auris Medical AG. H.S. received a one-time honorarium from the study sponsor for regulatory consulting work. T.M. is the Managing Director and a major shareholder of Auris Medical AG. The other authors declared no conflicts of interest. 


\section{References}

Adamchic I, Langguth B, Hauptmann C, Tass PA: Psychometric evaluation of visual analog scale for the assessment of chronic tinnitus. Am J Audiol 2012;21:215-225.

Baguley D, McFerran D, Hall D: Tinnitus. Lancet 2013;382:1600-1607.

Bird PA, Murray DP, Zhang M, Begg EJ: Intratympanic versus intravenous delivery of dexamethasone and dexamethasone sodium phosphate to cochlear perilymph. Otol Neurotol 2011;32:933-936.

Chan Y: Tinnitus: etiology, classification, characteristics, and treatment. Discov Med 2009;42 133-136.

Chen Z, Duan M, Lee H, Ruan R, Ulfendahl M: Pharmacokinetics of caroverine in the inner ear and its effects on cochlear function after systemic and local administrations in guinea pigs. Audiol Neurootol 2003;8:49-56.

DeMuro-Mercon C, McLeod L, Fehnel S, Meyer $\mathrm{T}$ : Determining clinically meaningful change in subjective tinnitus loudness. International Tinnitus Seminar, Berlin, May 2014

Dodson KM, Sismanis A: Intratympanic perfusion for the treatment of tinnitus. Otolaryngol Clin North Am 2004;37:991-1000.

Hall DA, Láinez MJ, Newman CW, Ganz Sanchez T, Egler M, Tennigkeit F, Koch M, Langguth B: Treatment options for subjective tinnitus: self reports from a sample of general practitioners and ENT physicians within Europe and the USA. BMC Health Serv Res 2011;11:302.

Hwang JH, Chen JC, Yang SY, Wang MF, Chan YC: Expression of tumor necrosis factor-a and interleukin- $1 \beta$ genes in the cochlea and inferior colliculus in salicylate-induced tinnitus. J Neuroinflammation 2011;8:30.
Kuk FK, Tyler RS, Russell D, Jordan H: The psychometric properties of a tinnitus handicap questionnaire. Ear Hear 1990;11:434-442.

Landgrebe M, Azevedo A, Baguley D, Bauer C, Cacace A, Coelho C, Dornhoffer J, Figueiredo R, Flor H, Hajak G, van de Heyning P, Hiller W, Khedr E, Kleinjung T, Koller M, Lainez JM, Londero A, Martin WH, Mennemeier M, Piccirillo J, De Ridder D, Rupprecht R, Searchfield G, Vanneste S, Zeman F, Langguth B: Methodological aspects of clinical trials in tinnitus: a proposal for an international standard. J Psychosom Res 2012;73:112-121.

Langguth B, Elgoyhen AB: Current pharmacological treatments for tinnitus. Expert Opin Pharmacother 2012;13:2495-2509.

Lewis S, Clarke M: Forest plots: trying to see the wood and the trees. BMJ 2001;322:14791480 .

Meikle MB, Stewart BJ, Griest SE, Henry JA: Tinnitus outcomes assessment. Trends Amplif 2008; 12:223-235.

Meyer T: Intratympanic treatment for tinnitus: a review. Noise Health 2013;15:83-90.

Moher D, Schulz KF, Altman D: The CONSORT statement: revised recommendations for improving the quality of reports of parallelgroup randomized trials. JAMA 2001;285: 1987-1991.

Muehlmeier G, Biesinger E, Maier H: Safety of intratympanic injection of AM-101 in patients with acute inner ear tinnitus. Audiol Neurotol 2011;16:388-397.
Nicolas-Puel C, Akbaraly T, Lloyd R, Berr C, Uziel A, Rebillard G, Puel JL: Characteristics of tinnitus in a population of 555 patients: specificities of tinnitus induced by noise trauma. Int Tinnitus J 2006;12:6470.

Puel JL, Ruel J, Guitton M, Wang J, Pujol R: The inner hair cell synaptic complex: physiology, pharmacology and new therapeutic strategies. Audiol Neurootol 2002;7:49-54.

Pujol R, Puel JL: Excitotoxicity, synaptic repair, and functional recovery in the mammalian cochlea: a review of recent findings. Ann N Y Acad Sci 1999;884:249-254.

Salt AN: Pharmacokinetics of drug entry into cochlear fluids. Volta Rev 2005; 105:277298.

Shargorodsky J, Curhan GC, Farwell WR: Prevalence and characteristics of tinnitus among US adults. Am J Med 2010;123:711-718.

Stouffer JL, Tyler RS: Characterization of tinnitus by tinnitus patients. J Speech Hear Disord 1990;55:439-453.

Tyler RS, Oleson J, Noble W, Coelho C, Ji H: Clinical trials for tinnitus: study populations, designs, measurement variables, and data analysis. Prog Brain Res 2007;266:499509.

van de Heyning P, Muehlmeier G, Cox T, Lisowska G, Maier H, Morawski K, Meyer T: Efficacy and safety of AM-101 in the treatment of acute inner ear tinnitus - a double-blind, randomized, placebo-controlled phase II study. Otol Neurotol 2014;35:589-597.

Vernon JA, Meikle MB: Tinnitus: clinical measurement. Otolaryngol Clin North Am 2003; 36:293-305. 